\title{
The role of the periaqueductal gray in escape behavior
}

\author{
Yaara Lefler $^{1 *}$, Dario Campagner ${ }^{1,2^{*}}$ and Tiago Branco ${ }^{1}$
}

${ }^{1}$ UCL Sainsbury Wellcome Centre for Neural Circuits and Behaviour

London W1T 4JG, UK

${ }^{2}$ UCL Gatsby Computational Neuroscience Unit

London W1T 4JG, UK

* Y. L. and D. C. contributed equally to this work

Corresponding author: t.branco@ucl.ac.uk 


\section{ABSTRACT}

Escape behavior is a defensive action deployed by animals in response to imminent threats. In mammalian species, a variety of different brain circuits are known to participate in this critical survival behavior. One of these circuits is the periaqueductal gray, a midbrain structure that can command a variety of instinctive behaviors. Recent experiments using modern systems neuroscience techniques have begun to elucidate the specific role of the periaqueductal gray in controlling escape. These have shown that periaqueductal gray neurons are critical units for gating and commanding the initiation of escape, specifically activated in situations of imminent, escapable threat. In addition, it is becoming clear that the periaqueductal gray integrates brainwide information that can modulate escape initiation to generate flexible defensive behaviors.

\section{INTRODUCTION}

When faced with a predator, animals adjust their defensive actions according to the perceived imminence of a potential attack. Threats that are distant are met with risk assessment and freezing behaviors, whereas those that are very close or approaching fast elicit escape behaviors such as running or jumping $[1,2]$. This hierarchical mapping of threat imminence to defensive behaviors implies that the brain has neural circuits for determining the threat level and linking it to the generation of specific actions. Accordingly, many brain regions have been associated with different aspects and levels of the entire action spectrum of defensive behavior. These include cortical, hypothalamic, midbrain and brainstem circuits, which together implement functions such as detecting and remembering threats, executing specific defensive actions and generating fear states $[3,4]$. For escape behavior, which is predominantly deployed in response to imminent threats, the periaqueductal gray (PAG) has long been known to be a key driving neural circuit. 
Seminal studies in the 1950s using electrical and chemical stimulation techniques revealed a functional topography of defensive actions within the PAG [5]. Stimulation of the dorsomedial and dorsolateral part of the PAG $(\mathrm{dPAG})^{1}$ generated active defensive actions such as jumping and running, while stimulation of the ventrolateral PAG (vlPAG) caused freezing and immobility [6,7]. Together with dPAG chemical inactivation and lesion experiments showing impairment of escape responses [5], these early studies placed the dPAG as a critical node for escape and opened the question of what its computational role is in generating escape during natural behavior.

Here we review recent studies in rodents and humans that have started to answer this question. Recordings from single dPAG neurons and optogenetic experiments in molecularly defined cell populations show high temporal correlation between the start of dPAG activity and escape onset, suggesting that the dPAG is responsible for commanding the initiation of escape behavior. Human fMRI studies substantiate this view by consistently showing PAG activation for threat stimuli that are imminent, suggesting that the PAG is specifically engaged to link immediate threat to escape actions. In addition, previous anatomical and histological work has shown that the dPAG receives afferents from many brain regions and contains receptors for a variety of neuromodulators [5,8]. Recent work has moved the field forward by manipulating specific pathways and neuromodulators to demonstrate their functional impact on escape behavior.

\footnotetext{
${ }^{1}$ While different functions have been attributed to dorsomedial, dorsolateral and lateral PAG [5], all these subdivisions have been implicated in escape behavior, and throughout this review we refer to them collectively as dPAG for simplicity.
} 


\section{IMMINENT THREAT AND ESCAPE INITIATION}

The dPAG is one of many neural circuits that participate in defensive behavior, and therefore a key question is what its role is in generating escape within this distributed network. From the outset, the dPAG seems well positioned to have a privileged role in linking imminent threat to escape initiation, as it receives input from sensory and limbic areas, and projects to centers that control locomotive movements, including the mesencephalic locomotor region $[8,9]$. Recent studies recording and manipulating activity of dPAG neurons seem to support this view. Work from our laboratory using calcium imaging in mice has shown that the activity of excitatory VGluT2+ dPAG neurons increases selectively during escape initiation [10]. Calcium signals in this neuronal population do not change during innately threatening stimuli that do not cause escape, and instead encode exclusively the choice to initiate escape (Figure 1A). This work further showed that information about threat is transmitted to the dPAG from the deep layers of the medial superior colliculus (dmSC) via weak and unreliable synapses, suggesting that only very salient and imminent threats generate enough synaptic activity to evoke action potentials in dPAG neurons. These data are compatible with a model where a population of dPAG excitatory neurons command the initiation of escape by thresholding threat evidence using a synaptic mechanism. In further support, electrophysiological recordings of single dPAG units in mice exposed to predators (rats) showed activity locked to the onset of instinctive flight in a subset of neurons [11,12]. Also, a large fraction of dPAG units in rats responded exclusively upon initiation of defensive movements in a fear conditioning paradigm [13].

Recent studies in humans also indicate that the PAG is predominantly activated in situations of imminent threats, especially when they are escapable. Building on previous fMRI work showing that the PAG is preferentially active during reactive fear - conditions of imminent danger that 
elicit fast reactions [14,15], Qi et al. [16] investigated fMRI signals while humans engaged in a computer-based task where they faced virtual predators that differed in the speed at which they attacked. The goal of the task was to minimize the loss of reward incurred from escaping, by fleeing as late as possible (Figure 1B - top panel). The authors found that while slow attacking predators activated mainly the hippocampus, posterior cingulate cortex, and prefrontal cortex, the decisions to escape from fast looming predators were correlated with activation of the PAG (Figure 1B - bottom panel) and midcingulate cortex. In agreement with these results, humans exposed to a paradigm where an approaching object signaled the timing of an electric shock showed increased PAG activity when the threat of shock was imminent [17]. PAG activation was greater when the shock was avoidable [17], and similar observations were made in a related threat escape task paradigm [18]. Further support for a PAG role in reacting to imminent threat comes from fMRI studies showing increased activation in situations where the threat is explicitly visible [19] and when threatening images loom fast in the direction of the participant [20]. Overall, the results from these human experiments argue for a conserved role of the PAG in processing imminent threat and initiating reactive escape actions. Future work using 7 tesla fMRI promises to take the field further by mapping defensive actions onto specific subdivisions of the human PAG [21].

Experiments manipulating the activity of PAG neurons have also substantiated their role in escape initiation. Optogenetic activation of excitatory dPAG neurons in mice causes flight responses with short latency [10,11,22], and the speed of escape scales positively with stimulation intensity, suggestive of a close interaction with the locomotor circuits that generate flight actions. This close link between dPAG population activity and the flight action is also supported by single unit recordings showing that dPAG activity correlates with, and precedes 
peak escape velocity $[11,12]$. One important observation arising from optogenetic activation of VGluT2+ dPAG neurons is that gradually increasing stimulation intensity causes an all-or-none increase in the probability of escape behavior [10]. This indicates that this population of excitatory VGluT2+ dPAG neurons initiates escape behavior but no other defensive actions. Accordingly, presenting imminent threats while optogenetically inhibiting these neurons causes freezing behavior [10]. This suggests a model where threat information is passed to both escape and freezing initiation centers, with escape being triggered specifically by the dPAG while inhibiting freezing initiation. When dPAG VGluT2+ neurons are inhibited, freezing can be expressed. It is important to note, however, that single unit recordings in the dPAG also reveal neurons that fire exclusively during risk assessment behavior [11,12]. As the neurons recorded are of unknown molecular identity, it is possible that dPAG cell populations other than VGluT2+ neurons integrate information about threats that are not imminent and that lead to behaviors lower in the defense hierarchy. These neurons could either participate in driving the behaviors themselves or help bring the dPAG population to the threshold for initiating escape as the threat becomes closer. In support of a wider role for $\mathrm{dPAG}$ neurons in generating defensive behavior, chemical and optogenetic stimulation experiments in rats could elicit not only active defense responses such as running and jumping, but also a progression from increased alertness and freezing with increasing stimulation intensity or frequency [23-25]. Similar to the single unit recording studies mentioned above, these stimulation experiments were done in molecularly undefined neurons, and therefore future experiments are needed to better dissect the wider role of multiple dPAG populations in controlling defensive behavior. Nevertheless, recent data are in support of a clear role of the dPAG in commanding the initiation of escape behavior in response to imminent threats, through a population of excitatory cells. 


\section{INTEGRATING BRAIN-WIDE INFORMATION TO MODULATE ESCAPE}

The PAG receives efferents not only from areas conveying information about imminent threats, such as the SC, but also from a vast array of cortical and subcortical circuits that potentially carry other types of information [26]. In addition, past work has demonstrated expression of receptors for a variety of neuromodulators across the PAG [5], which can modulate PAG-controlled behaviors, including active defense [27]. The key question is what information is transmitted by these circuit and neuromodulators to the escape PAG circuit, and what their role is in controlling escape behavior through their dPAG-projecting afferents. The most recent work in this area shows that many different PAG input pathways can act to positively or negatively modulate escape behavior, and thus confer a high degree of flexibility to escape, allowing it to adapt to a variety of internal and external states.

One of the major anatomical input pathways to the PAG is the hypothalamus [28]. The ventromedial hypothalamus (VMH) in particular, through its dorsomedial subdivision $(\mathrm{dmVMH})$, has been shown to be a key structure for regulating defensive behavior, and receives information about predator cues from the medial amygdala, mostly pheromonal and olfactory [4]. The dmVMH projects directly to the dPAG [28], and its chemogenetic inactivation decreases the expression of defensive behaviors [29]. However, in contrast with the dPAG, gradually increasing the strength of optogenetic stimulation of dmVMH excitatory cells in mice causes a gradual progression of behavior along the defensive hierarchy, with latencies in the order of seconds [30,31]. Recent single unit recordings in mice also suggest that VMH neurons gradually increase their firing rate during risk assessment as the distance to a predator decreases, while the activity profile of dPAG neurons resembles a thresholded version of VMH activity, showing firing rate increases only for proximal distances [12]. Together with more recent work showing 
persistent activation of dmVMH cells after threat exposure [32], these data suggest a model where the dmVMH integrates threat to generate a persistent state of fear or anxiety, which through $\mathrm{dPAG}$ projections can increase the drive to escape. While there is currently very limited information about the synaptic properties of the dmVMH-dPAG connection, the observation that dmVMH input to the dPAG does not seem to be enough to cause short latency escape on its own [30] suggests that this pathway might not be a driver connection for escape. Instead, dmVMHrecipient cells in the dPAG might integrate synaptic input from multiple sources, and thus the dmVMH may act mostly as a modulatory signal that requires additional coincident input to initiate escape (Figure 2A and B). Other hypothalamic inputs might serve similar functions, as indicated by recent work showing that inhibition of lateral hypothalamus neurons projecting to the PAG reduces the flight initiation distance, in agreement with an increase in the threshold for initiating escape [33]. A similar view is emerging for other PAG-projecting subcortical areas, such as the Zona Incerta, which is an inhibitory nucleus that can bi-directionally modulate the vigor of active defensive behavior [34].

Cortical projections to PAG provide a potential means for adding a layer of cognitive control to escape. A key candidate cortical area to serve this role is the medial prefrontal cortex (mPFC), which has been shown to project to $\mathrm{APAG}$ and mediate the behavioral expression of social defeat through decreasing dPAG activity [35]. Recent work recording from mPFC units shows responses selective for active avoidance [13], with the authors proposing that mPFC might contribute to regulate defensive strategies through PAG projections. In agreement with a role for mPFC in mediating behavior selection via PAG circuits, calcium imaging of mPFC-dPAG projecting neurons shows that activity increases in response to electric shocks, and decreases in response to reward [36]. In humans, the $\mathrm{mPFC}$ is active predominantly when the threat is distant 
and there is time for cognitive processing of the escape action, a period during which the PAG is silent $[15,16]$. More work is needed to understand how mPFC inputs act on the dPAG network to control escape behavior, and determine how they might contribute to behavioral selection. Another PAG-projecting cortical circuit with a role in arbitrating between defensive behaviors is the central amygdala (CeA, Figure 2C). Inhibitory CeA neurons can switch between active and passive conditioned defensive behaviors, through a projection to the vlPAG [22,37], a pathway that might be exploited by other circuits, such as the ventral tegmental area [38] and the pulvinar $[39,40]$. An interesting finding of this recent work is that the selection of freezing over escape behavior is implemented at the PAG level through disinhibition of the freezing pathway, and likely through simultaneous inhibition of the escape pathway [22]. This functional organization might well be an important principle of PAG function, whereby external input promotes a specific behavioral module, while intra-PAG connectivity implements a form of lateral inhibition to achieve clean selection. Beyond behavioral selection, cortical circuits are also well positioned to transmit to the dPAG sensory information important for escape behavior. The auditory cortex in particular has recently been shown to form a monosynaptic pathway with excitatory dPAG neurons, and modulating its activity has the ability to enhance and decrease sound-evoked escape behavior [41]. This pathway might be useful for complex or ambiguous sounds that may require cortical processing to establish stimulus identity, such as vocalizations, and future studies could test this hypothesis directly. An additional means of achieving flexibility of escape behavior through regulation of dPAG activity is neuromodulatory input [42-45]. Serotoninergic neurons in the dorsal raphe are a particularly interesting source of PAG modulation, as they are known to regulate avoidance behaviors [27,46], and have been recently found to be specifically active in situations of high threat levels, during which they promote escape behavior [47]. 


\section{CONCLUSION AND FUTURE DIRECTIONS}

An emerging model for the role of $\mathrm{dPAG}$ in escape behavior is that a population of dPAG neurons commands the initiation of escape actions in response to imminent threat. This has now been shown consistently by several studies through activity recordings and manipulations, and it seems to be a conserved property, from rodents to humans. One intriguing aspect of escape behavior is that it is specific for escapable threats and that the flight action can vary significantly depending on the threat context. An exciting future direction is to understand the mechanisms for linking dPAG activity selectively to escapable situations and to the optimal escape actions for each context. Possible models include the dPAG being selected by upstream contextual signals to provide a generic escape trigger, which is then refined into specific actions by additional contextual information. Alternatively, there could be several subpopulations of dPAG neurons capable of initiating different escape actions that are selected directly by contextual signals. A related and important aspect of dPAG function that should be addressed in future research is the role of dPAG neurons in other defensive behaviors, such as risk assessment. Are risk assessment dPAG neurons molecularly distinct from the ones that drive escape? Do they drive risk assessment behavior, or do they feed into escape cells to contribute to escape initiation?

One fundamental feature of the PAG is that it is a convergence point for a large array of neural circuits. Activity manipulations experiments show that cortical and subcortical inputs can act to modulate the initiation and vigor of escape to increase or decrease sensitivity to threatening stimuli. This provides a flexible link between threat and escape that can be adjusted to either veto or allow the expression of other behaviors depending on factors such as motivational states and experience. A key avenue of research will be to determine the mechanisms that control the selection of escape behavior over alternative defensive or appetitive actions. As new tools for 
imaging neuromodulator release become available [48], it will be particularly exciting to use them for understanding how different neuromodulatory states interact with the dPAG circuit to modulate escape behavior.

As an increasing number of brain circuits become linked to dPAG function and escape behavior, one of the most important challenges ahead is to understand how they work together during natural behavior to sculpt the expression of escape. In our opinion, the field should resist the temptation of being satisfied with the demonstration of necessity and sufficiency of particular circuit nodes, and instead should work towards understanding their specific contributions to the computation of escape. We believe that there is already enough knowledge about escape behavior and the underlying neural circuits to build possible models of how threat and experience generate escape. These models and the predictions they make should guide the design of future experiments.

\section{ACKNOWLEDGEMENTS}

We thank V. Stempel, R. Vale and the editors of this issue for comments on the manuscript, G. Costa for artwork. This work was supported by Wellcome [214352/Z/18/Z], Gatsby Charitable Foundation, and European Union's Horizon 2020 Marie Skłodowska-Curie [706136].

\section{REFERENCES}

1. Blanchard DC, Litvin Y, Pentkowski NS, Blanchard RJ: Defense and Aggression. In 
Handbook of Neuroscience for the Behavioral Sciences. John Wiley \& Sons, Inc.; 2009.

2. Fanselow MS, Lester LS: A functional behavioristic approach to aversively motivated behavior: Predatory imminence as a determinant of the topography of defensive behavior. In Evolution and learning . Edited by Bolles RC, Beecher MD. Lawrence Erlbaum Associates, Inc. ; 1988:185-212.

3. Tovote P, Fadok JP, Lüthi A: Neuronal circuits for fear and anxiety. Nat Rev Neurosci 2015, 16:317-331.

4. Gross CT, Canteras NS: The many paths to fear. Nat Rev Neurosci 2012, 13:651-658.

5. Depaulis A, Bandler R: The Midbrain Periaqueductal Gray Matter: Functional, Anatomical, and Neurochemical Organization (NATO ASI Series). 1990.

6. Bandler R, Carrive P: Integrated defence reaction elicited by excitatory amino acid microinjection in the midbrain periaqueductal grey region of the unrestrained cat. Brain Res 1988, 439:95-106.

7. Fanselow MS, DeCola JP, De Oca BM, Landeira-Fernandez J: Ventral and Dorsolateral Regions of the Midbrain Periaqueductal Gray (PAG) Control Different Stages of Defensive Behavior. Aggress Behav 1995, 21:63-77.

8. Marchand JE, Hagino N: Afferents to the periaqueductal gray in the rat. A horseradish peroxidase study. Neuroscience 1983, 9:95-106.

9. Ferreira-Pinto MJ, Ruder L, Capelli P, Arber S: Connecting Circuits for Supraspinal Control of Locomotion. Neuron 2018, 100:361-374.

10. Evans DA, Stempel AV, Vale R, Ruehle S, Lefler Y, Branco T: A synaptic threshold mechanism for computing escape decisions. Nature 2018, 558:590-594.

11. Deng H, Xiao X, Wang Z: Periaqueductal Gray Neuronal Activities Underlie Different Aspects of Defensive Behaviors. J Neurosci 2016, 36:7580-7588.

12. Masferrer ME, Silva BA, Nomoto K, Lima SQ, Gross CT: Differential encoding of predator fear in the ventromedial hypothalamus and periaqueductal grey. bioRxiv 2018, doi:10.1101/283820.

13. Halladay LR, Blair HT: Distinct ensembles of medial prefrontal cortex neurons are activated by threatening stimuli that elicit excitation vs. inhibition of movement. $J$ Neurophysiol 2015, 114:793-807.

14. Mobbs D, Marchant JL, Hassabis D, Seymour B, Tan G, Gray M, Petrovic P, Dolan RJ, Frith CD: From threat to fear: The neural organization of defensive fear systems in humans. J Neurosci 2009, 29:12236-12243.

15. Mobbs D, Petrovic P, Marchant JL, Hassabis D, Weiskopf N: When Fear Is Near: Threat Imminence Elicits Prefrontal- Periaqueductal Gray Shifts in Humans. Science (80- ) 2007, 1119:1079-1083.

16. Qi S, Hassabis D, Sun J, Guo F, Daw N, Mobbs D: How cognitive and reactive fear circuits optimize escape decisions in humans. Proc Natl Acad Sci 2018, 
115:201712314.

17. Wendt J, Löw A, Weymar M, Lotze M, Hamm AO: Active avoidance and attentive freezing in the face of approaching threat. Neuroimage 2017, 158:196-204.

18. Terburg D, Scheggia D, Triana del Rio R, Klumpers F, Ciobanu AC, Morgan B, Montoya ER, Bos PA, Giobellina G, van den Burg EH, et al.: The Basolateral Amygdala Is Essential for Rapid Escape: A Human and Rodent Study. Cell 2018, 175:723-735.e16.

19. Rigoli F, Ewbank M, Dalgleish T, Calder A: Threat visibility modulates the defensive brain circuit underlying fear and anxiety. Neurosci Lett 2016, 612:7-13.

20. Coker-Appiah DS, White SF, Clanton R, Yang J, Martin A, Blair RJR: Looming animate and inanimate threats: The response of the amygdala and periaqueductal gray. Soc Neurosci 2013, 8:621-630.

21. Faull OK, Jenkinson M, Clare S, Pattinson KTS: Functional subdivision of the human periaqueductal grey in respiratory control using 7 tesla fMRI. Neuroimage 2015, 113:356-364.

22. Tovote P, Esposito MS, Botta P, Chaudun F, Fadok JP, Markovic M, Wolff SBE, Ramakrishnan C, Fenno L, Deisseroth K, et al.: Midbrain circuits for defensive behaviour. Nature 2016, 534:206-212.

23. Chen SC, Zhou H, Guo SC, Zhang JC, Qu Y, Feng ZY, Xu K Di, Zheng XX: Optogenetics Based Rat-Robot Control: Optical Stimulation Encodes "Stop" and "Escape" Commands. Ann Biomed Eng 2015, 43:1851-1864.

24. Assareh N, Sarrami M, Carrive P, McNally GP: The organization of defensive behavior elicited by optogenetic excitation of rat lateral or ventrolateral periaqueductal gray. Behav Neurosci 2016, 130:406-14.

25. Ullah F, dos Anjos-Garcia T, dos Santos IR, Biagioni AF, Coimbra NC: Relevance of dorsomedial hypothalamus, dorsomedial division of the ventromedial hypothalamus and the dorsal periaqueductal gray matter in the organization of freezing or oriented and non-oriented escape emotional behaviors. Behav Brain Res 2015, 293:143-152.

26. Marchand JE, Hagino N: Afferents to the periaqueductal gray in the rat. A horseradish peroxidase study. Neuroscience 1983, 9:95-106.

27. Shaikh MB, De Lanerolle NC, Siegel A: Serotonin 5-HT1A and 5-HT2/1C receptors in the midbrain periaqueductal gray differentially modulate defensive rage behavior elicited from the medial hypothalamus of the cat. Brain Res 1997, 765:198-207.

28. Hahn JD, Sporns O, Watts AG, Swanson LW: Macroscale intrinsic network architecture of the hypothalamus. Proc Natl Acad Sci 2019, 116:8018-8027.

29. Silva BA, Mattucci C, Krzywkowski P, Murana E, Illarionova A, Grinevich V, Canteras NS, Ragozzino D, Gross CT: Independent hypothalamic circuits for social and predator fear. Nat Neurosci 2013, 16:1731-3.

30. Wang L, Chen IZ, Lin D: Collateral Pathways from the Ventromedial Hypothalamus 
Mediate Defensive Behaviors. Neuron 2015, 85:1344-1358.

31. Kunwar PS, Zelikowsky M, Remedios R, Cai H, Yilmaz M, Meister M, Anderson DJ: Ventromedial hypothalamic neurons control a defensive emotion state. Elife 2015, 4.

32. Kennedy A, Kunwar PS, Li L, Wagenaar D, Anderson DJ: Stimulus-specific neural encoding of a persistent, internal defensive state in the hypothalamus Ann. BioRxiv 2019 ,

33. Li Y, Zeng J, Zhang J, Yue C, Zhong W, Liu Z, Feng Q, Luo M: Hypothalamic Circuits for Predation and Evasion. Neuron 2018, 97:911-924.e5.

34. Chou XL, Wang X, Zhang ZG, Shen L, Zingg B, Huang J, Zhong W, Mesik L, Zhang LI, Tao HW: Inhibitory gain modulation of defense behaviors by zona incerta. Nat Commun 2018, 9:1-12.

35. Franklin TB, Silva BA, Perova Z, Marrone L, Masferrer ME, Zhan Y, Kaplan A, Greetham L, Verrechia V, Halman A, et al.: Prefrontal cortical control of a brainstem social behavior circuit. Nat Neurosci 2017, 20:260-270.

36. Vander Weele CM, Siciliano CA, Matthews GA, Namburi P, Izadmehr EM, Espinel IC, Nieh EH, Schut EHS, Padilla-Coreano N, Burgos-Robles A, et al.: Dopamine enhances signal-to-noise ratio in cortical-brainstem encoding of aversive stimuli. Nature 2018, 563:397-401.

37. Fadok JP, Krabbe S, Markovic M, Courtin J, Xu C, Massi L, Botta P, Bylund K, Müller C, Kovacevic A, et al.: A competitive inhibitory circuit for selection of active and passive fear responses. Nature 2017, 542:96-99.

38. Zhou Z, Liu X, Chen S, Zhang Z, Liu Y, Montardy Q, Tang Y, Wei P, Liu N, Li L, et al.: A VTA GABAergic Neural Circuit Mediates Visually Evoked Innate Defensive Responses. Neuron 2019, doi:10.1016/j.neuron.2019.05.027.

39. Shang C, Liu Z, Chen Z, Shi Y, Wang Q, Liu S, Li D, Cao P: A parvalbumin-positive excitatory visual pathway to trigger fear responses in mice. Science (80- ) 2015, 348:1472-1477.

40. Wei P, Liu N, Zhang Z, Liu X, Tang Y, He X, Wu B, Zhou Z, Liu Y, Li J, et al.: Processing of visually evoked innate fear by a non-canonical thalamic pathway. Nat Commun 2015, 6:1-12.

41. Wang H, Chen J, Xu X, Sun W, Chen X, Zhao F, Luo M-H, Liu C, Guo Y, Xie W, et al.: Direct auditory cortical input to the lateral periaqueductal gray controls sounddriven defensive behavior. PLOS Biol 2019, 17:e3000417.

42. Fernandes GG, Frias AT, Spiacci A, Pinheiro LC, Tanus-Santos JE, Zangrossi H: Nitric oxide in the dorsal periaqueductal gray mediates the panic-like escape response evoked by exposure to hypoxia. Prog Neuro-Psychopharmacology Biol Psychiatry 2019, 92:321-327.

43. Zhou P, Xu H-S, Li M-M, Chen X-D, Wang J, Zhou H-B, Chen L, Zhang N, Liu N: Mechanism of nitric oxide and acid-sensing ion channel 1a modulation of panic-like 
behaviour in the dorsal periaqueductal grey of the mouse. Behav Brain Res 2018, 353:32-39.

44. Fiaes GC de S, Roncon CM, Sestile CC, Maraschin JC, Souza RLS, Porcu M, Audi EA: Panicolytic-like effect of tramadol is mediated by opioid receptors in the dorsal periaqueductal grey. Behav Brain Res 2017, 326:52-58.

45. Batista LA, Bastos JR, Moreira FA: Role of endocannabinoid signalling in the dorsolateral periaqueductal grey in the modulation of distinct panic-like responses. $J$ Psychopharmacol 2015, 29:335-43.

46. Zanoveli JM, Nogueira RL, Zangrossi H: Serotonin in the dorsal periaqueductal gray modulates inhibitory avoidance and one-way escape behaviors in the elevated $\mathrm{T}$ maze. Eur J Pharmacol 2003, 473:153-61.

47. Seo C, Guru A, Jin M, Ito B, Sleezer BJ, Ho Y-Y, Wang E, Boada C, Krupa NA, Kullakanda DS, et al.: Intense threat switches dorsal raphe serotonin neurons to a paradoxical operational mode. Science 2019, 363:538-542.

48. Patriarchi T, Cho JR, Merten K, Howe MW, Marley A, Xiong W-H, Folk RW, Broussard GJ, Liang R, Jang MJ, et al.: Ultrafast neuronal imaging of dopamine dynamics with designed genetically encoded sensors. Science 2018, 360:eaat4422. 
A

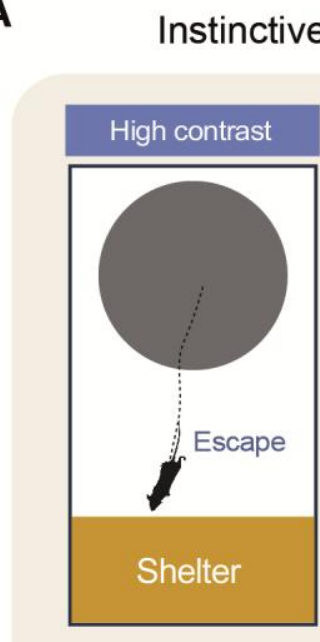

escape in mice

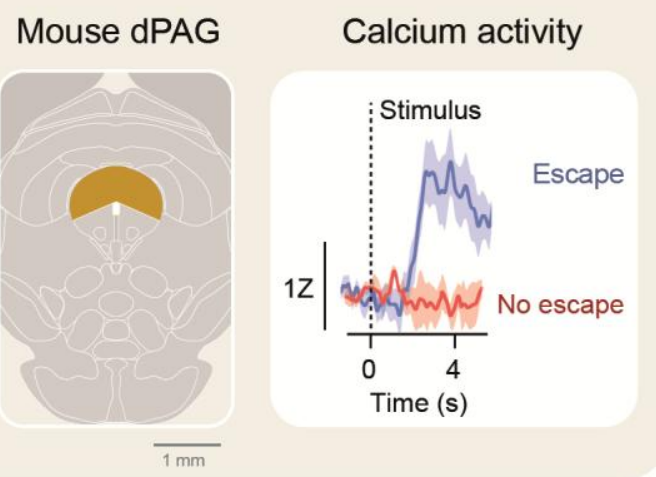

B Escape from virtual predators in humans
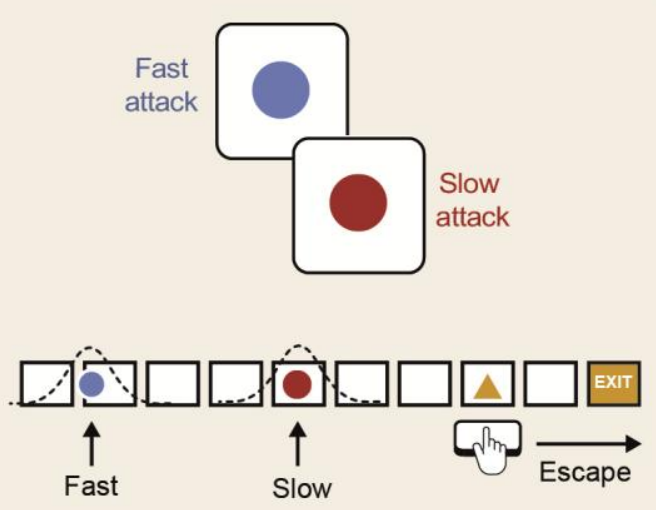

Human PAG fMRI activity

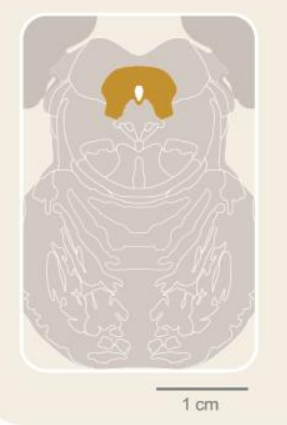

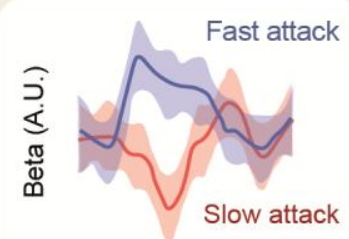

0

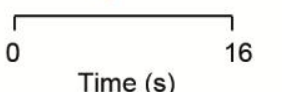

Figure 1. PAG neurons are active during escape initiation from imminent threats in rodents and humans.

(A) Activation of mouse dPAG during initiation of escape behavior. Schematic representation of a mouse responding to innately aversive overhead expanding spots of different contrasts (top). High contrast spots elicit escape and increase the activity of a population of excitatory dPAG neurons (bottom), whereas low contrast spots fail to cause escape and dPAG activity. Dotted lines illustrate mouse trajectory from stimulation onset, and dPAG activity profiles are from calcium imaging data. Adapted from [10]. Source of mouse silhouette: https://scidraw.io/.

(B) Selective activation of human PAG in response to imminent threat. Schematic of a behavioral assay for studying escape behavior in humans (top). Subjects are presented with the image of a virtual predator that moves toward the subject (represented by a triangle), and the predator color signal how fast it attacks. The subject can escape by pressing a button before the predator attacks and is given a reward if it succeeds, or an aversive electric shock is it fails. Only escape from fast attacking predators causes activation of the PAG during fMRI (bottom). The 
right panel shows the evolution of the beta parameter predicting the hemodynamic signal in the PAG from trial type (fast or slow attack). Adapted from [16]. Brain schematics have been adapted from Allen Mouse Brain Atlas and Allen Human Brain Atlas.

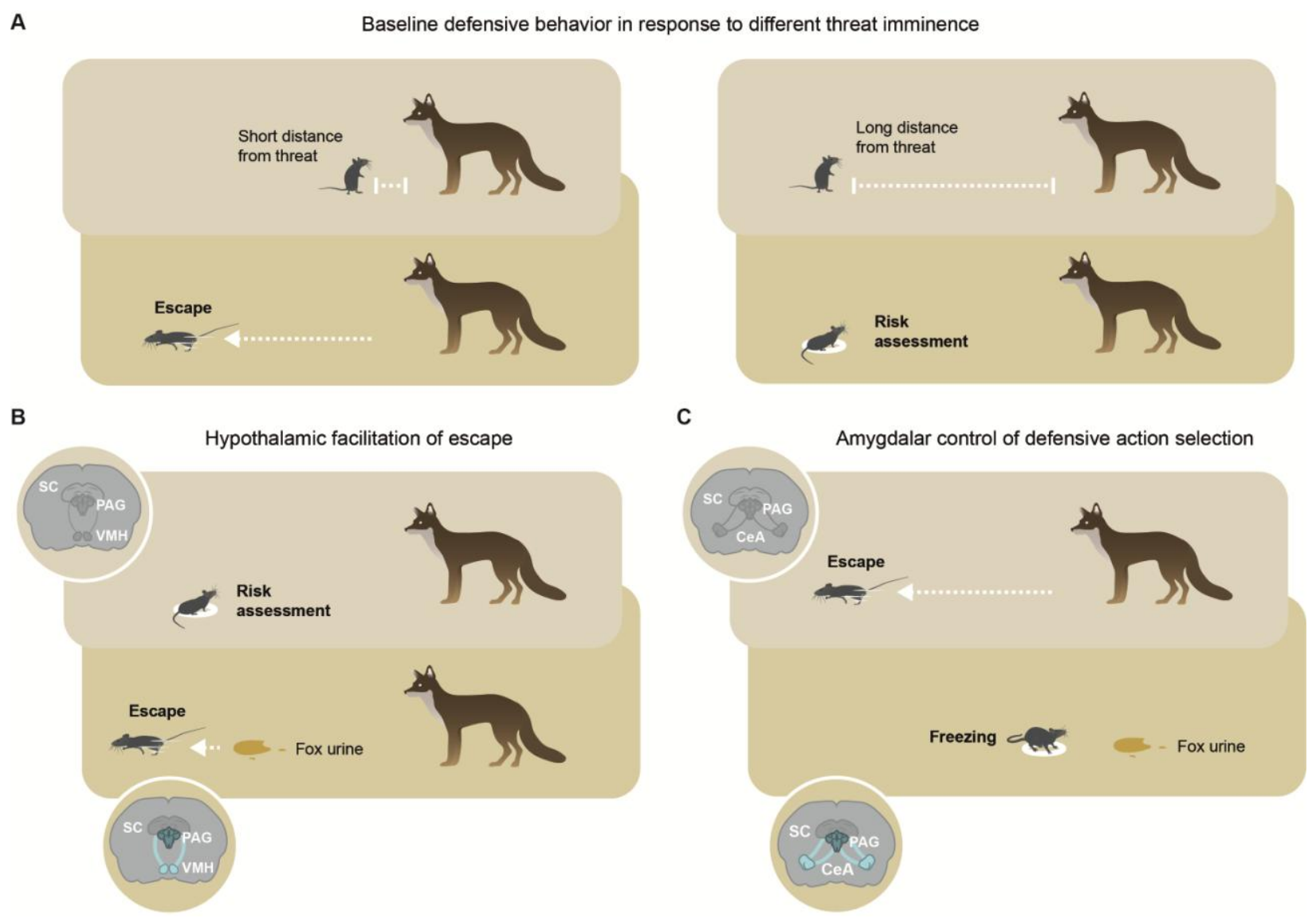

\section{Figure 2. Cortical and subcortical projections to PAG modulate escape behavior.}

(A) Animals adjust their defensive strategy to the perceived threat imminence. Mice faced with a predator in their immediate proximity initiate fast escape behaviors, such as running (left), whereas the perception of a distant predator triggers more passive defensive behaviors, such as risk assessment (right).

(B) PAG-projecting VMH neurons modulate escape behavior by integrating pheromonal and olfactory cues. Risk assessment behavior in response to distant threats (top) can be converted into escape by integration of additional cues (bottom), such as predator odors (e.g.: fox urine). Activation of PAG-projecting VMH neurons (blue) promotes a gradual progression of behavior along the defensive hierarchy and increases the flight initiation distance. 
(C) PAG-projecting CeA neurons can switch between active and passive defensive behaviors. Proximal encounters with a predator can lead to an aversive memory associated with predator cues. Subsequent exposure to these conditioned cues can lead to freezing behavior, even in the absence of the predator itself, which is gated by PAG-projecting CeA neurons (bottom, blue). 